\title{
Utilization of Art Therapy with Children with ADHD Co-Morbid Intellectual Disability
}

\section{for Decreasing Inattention}

* Dr. Hina Ayaz Habib, Assistant Professor (Corresponding Author)

$$
\text { ** Dr. Uzma Ali }
$$

\begin{abstract}
The research aimed to examine the positive role art therapy could play in improving the attention span of children with Attention-Deficit/Hyperactivity Disorder co-morbid Intellectual Disability in the context of Karachi (Pakistan). The key presumption was that art therapy can help in decreasing inattention among the children with ADHD co-morbid Intellectual Disability. Purposive sampling was used for the selection of cases from schools in Karachi (Pakistan) which are offering educational services to the children with special needs. The sample comprised 14 children, ages 6 to 12 years (mean age $=7.391 ; S D=1.315$ ) with ADHD co-morbid Mild ID. Experimental design was adopted for carrying out the investigation, entailing the division of the sample into the treatment group (which received 25 art therapy sessions as a form of intervention) and control group (which was provided with regular classroom activities). The allocation of children to both groups was carried out through the ABBA technique as well as the use of observation. The tools of the Attention Deficit Hyperactivity Disorder test were used to gauge the differences in the level of inattention in both groups. Analysis of data was conducted through SPSS version 17.0, using descriptive statistics to examine the pattern of data as well as identify the level of effectiveness of art therapy through administering the Wilcoxon test. The findings of the study support the assumption that art therapy is useful for curtailing impulsiveness in children with ADHD. Therefore, it can be concluded that art therapy is likely to bring positive changes in the level of attention in children with ADHD co-morbid ID. The study carries implications for individuals practicing in the field of mental health care and special education.
\end{abstract}

Keywords: Inattention, ADHD Co-morbid Intellectual Disabilities (ID), Art Therapy. Introduction

Attention Deficit Hyperactivity Disorder (ADHD) is a widespread neurobiological disorder that causes hindrance in the academic and social functioning of $3 \%$ to $5 \%$ of school-aged children (MTA Cooperative Group, 1999). Even though the symptoms of ADHD can vary, it is marked by three core features: impulsivity, hyperactivity, and inattention which can negatively affect the day to day functioning of an individual (Biederman, 2005). The symptoms of ADHD are commonly identified during childhood, with some people experience persistent symptoms during adulthood as well.

Impulsivity and hyperactivity as characteristics of ADHD are evident in the form of actions lacking foresight as well as reflecting the need for instant satisfaction, which often becomes a source of harm for the individual. The harm not necessarily pertains to physical damage, but also involves damage to social relationships (American Psychiatric Association, 2013). Inattention can manifest in the form of various behaviors, such as wandering away from the task at hand and a sustained inability to maintain focus on the task at hand (Arns, de Ridder, Strehl, Breteler \& Coenen, 2009). The symptom of inattention arises out of the problematic cognitive processing and functioning (American Psychiatric Association, 2013).

Various approaches have been adopted to help children cope with the problems associated with ADHD, with each treatment method carrying the potential efficacy in decreasing the harm caused by ADHD. The treatment options adopted for treating ADHD symptoms include psychopharmacology, psycho-social interventions (Pliszka, 2009), and even music therapy aiming to reduce the dysfunctional consequences of ADHD (Jackson, 2003). Antshel, Hargrave, Simonescu,

* Institute of Clinical Psychology, University of Karachi, Pakistan Email: drhinaayazhabib@uok.edu.pk

** Institute of Clinical Psychology, University of Karachi Email: uzma_kamranali@yahoo.com 
Kaul, Hendricks, and Faraone (2011) have indicated that Cognitive Behavioral Therapy (CBT) can help individuals with ADHD in overcoming the issues of impulsivity and inattention. In the case of children, MTA Cooperative Group (1999) has used a Multimodal treatment approach, offering the children with psychopharmacology and/or psycho-social based intervention. It was found that combined treatment held greater efficacy in the treatment of ADHD as compared to the use of a single treatment option. Earlier scholars have established the effectiveness of behavior therapy for children with ADHD, considering it to be a suitable course of treatment (Carlson, Pelham Jr, Milich \& Dixon, 1992; Klein \& Abikoff, 1997).

Apart from the behavior-based therapy, art therapy (AT) is gaining significance as one of the contemporary treatment options for managing ADHD symptoms. The key purpose of art therapy is to help the individual in healing while using the medium of art as the means of facilitating the process. The majority of the researches conducted on the efficacy of AT art therapy has focused on its beneficial effects on different socio-emotional behaviors (Freilich \& Shechtman, 2010, Slayton, D'Archer, \& Kaplan, 2010). AT helps children experiencing psychosocial problems arising out of ADHD, providing them a medium through which they can adequately express themselves. Thus, promoting positive behaviors and increased degree of behavioral control among them (Henley, 1998).

According to the findings of the study Safran (2002) conducted with Children suffering from ADHD using AT, AT was found to be an invaluable intervention as it can offer the psychotherapist with the pre-intervention status of the child thus help in maintaining track of the changes and growth occurring through the utilization of the intervention. At the same time, children suffering from sensory integration difficulties can significantly advantage from regular sessions based on AT. AT sessions can facilitate children with ADHD in social integration and make them more focused (Kearns, 2014). A related standpoint has been assumed for the current study, using "art as therapy".

In light of the above researches, it can be argued that AT can serve as a useful tool for decreasing the problems of impulsiveness and inattention which are core features of ADHD. There is some evidence suggesting the beneficial impact of art therapy for bringing desirable behavioral change among children dealing with various problems (Epp, 2008; Kozlowska \& Hanney, 2001; Waller, 2006). Some researches attribute reduction in impulsive behavior and inattention to different forms of art therapy such as mandala drawing (Smitheman-Brown \& Church, 1996). However, there is a need to further explore the usefulness of AT in dealing with the cognitive-behavioral symptoms of children with ADHD.

Based on the literature discussed earlier, the current study will investigate how effective art therapy is for children with ADHD comorbid with Intellectual Disability. The key assumption is that art therapy holds significance in the treatment of impulsive behavior and inattention for children with ADHD co-morbid ID. The hypothesis drawn out of this context presumes that children offered art therapy as a treatment will show a notable decrease in inattention as compared to the control group that was offered no treatment.

\section{Method}

\section{Participants}

The sample included 14 children (6 to 12 years; mean= 7.391; SD =1.315) with ADHD co-morbid ID who were selected from Sindh Special Education Center: Qauiden, Karachi, Pakistan. The selection was made based on qualifying for the diagnostic criteria of ADHD (mild) on Axis I and Mild intellectual disability on Axis II DSM-IV. Clinical Interview, assessment as well as the criteria given in DSM-IV was referred to reach the diagnosis of ADHD. ABBA technique was used for the distribution of the sample into the experimental group ( $\mathrm{n}=7 ; 3$ girls and 4 boys) and control group $(\mathrm{n}=7 ; 2$ girls and 5 boys) (refer to table 1$)$.

\section{Measures}

\section{Demographic Data Sheet}

It included the child's name, age, gender, birth order, and year of schooling. The sheet also contained questions related to parents, parental qualification, per year income, profession, and family structure.

\section{Observation Recording Sheet}

The recording sheet contained information about significant behaviors, verbalization, and procedures used while conducting the art therapy sessions. It provided information in terms of baseline data and the progression of the child. 


\section{Attention-Deficit / Hyperactivity Disorder Test (ADHD-T)}

The ADHDT was developed by Gilliam (1995), using the criterion of ADHD as depicted in DSM-IV. The test measures three aspects of ADHD through the subtests namely: hyperactivity, Impulsivity, and Inattention using 36 items. The subtests have a significant level of internal consistency and reliability as manifested through the scores of .80s and .90s (Gilliam, 1995). The test provides raw scores, ADHD Quotient, and the percentile. For the current study, ADHD-T was administered on the sample and their raw scores were used.

\section{Procedure}

The director of the school and teachers were approached to seek permission for the study and obtain informed consent from them. The informed consent form was presented to the director and concerned teachers providing information about the purpose of the study and the treatment method to be used in the investigation. Once permission was granted the process of data collection was initiated.

The researcher has adopted an experimental design, dividing the sample into an experimental and control group. The children in the experimental group were recipients of art therapy sessions while the children included in the control group were only offered the regular school-based activities. The division of the sample into two groups allowed the comparison of the effect of art therapy on inattention. After the art therapy sessions were concluded, the level of inattention of the experimental and control group was measured.

\section{Pre Intervention}

Before the initiation of art therapy sessions, the children included in the sample were observed for 2 days for 8 hours to identify the behavioral patterns associated with the ADHD. This provided a benchmark for comparison of the resultant changes due to AT sessions. After the observation, ADHD-T was administered by the researcher, which provided the researcher with information about the magnitude and frequency of inattention experienced by the children with ADHD. In the first session, the children were allowed to draw freely which helped in identifying their artistic skills and developmental level, which supported the researcher in devising suitable goals. The framework of Malchiodi (2003) was adopted for comprehending the degree of artistic competence of the children with ADHD included in the study. The pre-intervention phase has facilitated the development of a baseline for comparison.

\section{Intervention phase}

During the intervention phase, the researcher has used the techniques of behavior therapy, specifically, Roth's therapeutic framework was followed as a structure for conducting art therapy sessions. Positive reinforcement such as verbal appreciation as well as non-verbal means of appreciation was used when the children depicted favorable behavior patterns. The encouragement children received through positive reinforcement increases the likelihood of repetition of those behaviors and created a foundation for the therapeutic alliance. The researcher has used modeling, shaping, and prompting during the AT session and offered the children with pre-art materials and materials that could hold their interest and encouraged them to play an active part during the sessions.

During the sessions, the artwork of children was kept in a specific folder designated to a child, organized with the key information about the session such as session number, date on which the AT session was held, and the name of the child (coded). Along with that, the researcher has accumulated the observations of the child's behavior, verbalization, and questions (case notes) during each session, which provided a useful source of information about the process during each session.

\section{Post-intervention}

During the post-intervention phase, a comparison of pre and post levels of inattention was carried out by using ADHD-T. During the sessions, the artwork that children have made and case notes provided the foundation of comparison of inattentive behavior among children with ADHD.

\section{Statistical Analysis}

The statistical analysis of data was carried out using SSPS Vol 17. Descriptive tests (mean, standard deviation, frequency, and percentages) were calculated to illustrate the demographic features of the data. Wilcoxon test for difference was identified as the suitable inferential statistics technique.

\section{Results}

Table 1 shows the mean and standard deviation of the sample and the distribution of samples into the control and experimental group. 
Table 1. Mean and standard deviation of age of children according to groups.

\begin{tabular}{lccc}
\hline Groups & N & Mean & SD \\
\hline Age of children & 14 & 7.391 & 1.315 \\
Control ADHD (ID) & 7 & 7.70 & 1.3317 \\
Treatment ADHD (ID) & 7 & 7.543 & 1.1178 \\
\hline
\end{tabular}

Table 2 indicates that their art therapy had a significantly positive influence on the level of attention of the children with ADHD, as the level of inattention had decreased after treatment.

Table 2 Comparison of treatment and control group of children with ADHD comorbid ID on inattention before and after treatment $(n=14)$.

\begin{tabular}{|c|c|c|c|c|c|c|}
\hline \multirow[b]{2}{*}{ Groups } & \multirow[b]{2}{*}{$\mathrm{N}$} & \multicolumn{2}{|c|}{ Inattention Pre-treatment } & \multicolumn{2}{|c|}{ Inattention Post-treatment } & \multirow[b]{2}{*}{$\mathrm{P}$} \\
\hline & & Median & Range & Median & Range & \\
\hline $\begin{array}{l}\text { Control ADHD } \\
\text { with ID }\end{array}$ & 7 & 20 & $16-24$ & 20 & $17-24$ & 0.317 \\
\hline $\begin{array}{l}\text { Treatment } \\
\text { ADHD with ID }\end{array}$ & 7 & 15 & $13-18$ & 14 & $12-17$ & $0.014 *$ \\
\hline
\end{tabular}

Note $(\mathrm{p}<.05) *$ Wilcoxon signed ranked test

\section{Discussion}

The efficacy of art therapy as a mode of treating children with behavioral and cognitive problems has been emphasized by various researchers (Jackson, 2003; Webster-Stratton, Reid \& Hammond, 2001). Schweizer, Knorth, and Spreen (2014) reviewed the literature published in the last 30 years related to AT and ASD. After reviewing 18 publications they summarized that children after receiving sessions of AT experience higher self-confidence and become better at regulating their emotions. Mousavi and Sohrabi (2014) studied 30 children who had anger and aggressiveness problems and found that, after a 10 -week art therapy program, these children showed less anger and increase levels of self-esteem. The results obtained in this study illustrate that art therapy not only holds relevance to the needs of the children with ADHD comorbid ID but also plays a significant role in helping them in overcoming the issue of inattention. A similar finding is evident in the work of Safran (2003) who viewed it as a suitable medium of expression for children. Since AT is dependent on the use of visual learning skills, children are likely to find it easier to express themselves. It has been further asserted that the activities adopted by AT are specifically child-centered, thus making it an appropriate choice for treating the problems faced by children with ADHD. Besides, Safran (2003) has noted that art therapy has relevance for children experiencing developmental delays as well.

Even though there is limited evidence available which indicates art therapy an effective mode of treatment, the available researches has established its effectiveness for minimizing the negative effect of ADHD. Association of Natural Psychology (2006) has concluded that AT brings stability in the lives of children with ADHD, thus promoting an increase in the level of attention while lowering the degree of impulsivity. Children with ADHD can learn to deal with delays and adjust with the notion of delayed gratification without their symptoms become aggravated. The experimental group of the current study has demonstrated decreased inattention (refer to table 2) which supports the idea that the children with ADHD can learn to slow down and bring consistency in their behaviors with the help of AT.

In a similar study, Smitheman-Brown and Church (1996) have engaged children in the creation of mandala drawings during AT sessions and found it to be strongly associated with positive cognitive and behavioral changes. The cognitive changes were evident in the form of improved attention span and healthier decision making patterns, while behavioral changes were reflected in the form of the ability to stay focused on the task at hand and its successful completion.

Researchers have also claimed art therapy to be beneficial for bringing positive changes in the moor of an individual (Dalebroux, Goldstein \& Winner, 2008; Drake, Coleman \& Winner, 2011). Moreover, Abbott, Shanahan, and Neufeld (2013) have stated that engagement in art-related and creative activities helps in lowering the level of stress of an individual. Keeping this context into consideration, the effectiveness of AT for cognitive, behavioral, and affective improvement becomes more profound.

\section{Conclusion and Future Directions}

The medium of art therapy has enabled the children to express themselves uniquely, sharing their individuality with the researcher while providing insight into the way ADHD limits their functioning. 
ADHD has a profound effect on the cognitive and behavioral aspects which in turn gives rise to psycho-social problems such as inability to concentrate, poor attention, and impulsiveness. Art therapy helps children with ADHD express their feelings through the medium of art, providing the therapist with a context to create a beneficial set up for therapy. Therefore, it can be concluded that art therapy helps in reducing the issue of inattention among children with ADHD and develops a positive relationship between the therapist and child.

Future researchers can focus on the role art therapy can play in shaping the creativity of children with ADHD, using a combination of neurobiological and behavioral perspectives to uncover the wider scope of benefits of AT. Since the current study provides foundations for understanding the usefulness of art therapy in Pakistan, future researchers can further explore this area and its therapeutic effectiveness for children with ADHD. The findings of the study suggest that AT can be applied as a part of classroom activities in schools to help children in coping with the distress caused by ADHD symptoms. Special education schools can specifically benefit from the integration of AT in the school activities, facilitating teachers to gain an understanding of the challenges faced by children with ADHD and helping them in coping with these issues.

\section{Ethical Consideration}

Informed consent was obtained from the institute's director and teachers before the collection of information from the respondents. Besides, the confidentiality and anonymity of the children included in the study were maintained as the researcher has used pseudonyms instead of actual names of children.

\section{Acknowledgment}

The researcher is grateful to Kishwer Sultan, Director Sindh Special Education Center: Qauiden, and the teachers who have provided huge support in conducting the study. Accessing the participants and their file record was made possible by them. The researcher would also like to thank parents for providing permission for the inclusion of their children in the study. The researcher is also thankful to the children who have participated in the study and cooperated in the process of data collection.

\section{References}

Abbott, K. A., Shanahan, M. J., \& Neufeld, R. W. (2013). Artistic tasks outperform nonartistic tasks for stress reduction. Art Therapy, 30(2), 71-78.

American Psychiatric Association. (2013). Diagnostic and statistical manual of mental disorders (5th ed.). Washington, DC: American Psychiatric Publishing.

Antshel, K. M., Hargrave, T. M., Simonescu, M., Kaul, P., Hendricks, K., \& Faraone, S. V. (2011). Advances in understanding and treating ADHD. BMC medicine, 9(1), 72-83.

Arns, M., de Ridder, S., Strehl, U., Breteler, M., \& Coenen, A. (2009). Efficacy of neurofeedback treatment in ADHD: the effects on inattention, impulsivity, and hyperactivity: a metaanalysis. Clinical EEG and neuroscience, 40(3), 180-189.

Association for Natural Psychology (2006). Art Therapy. Retrieved from http://www.winmentalhealth.com/arts_therapy.php

Biederman, J. (2005). Attention-deficit/hyperactivity disorder: a selective overview. Biological psychiatry, 57(11), 1215-1220.

Carlson, C. L., Pelham Jr, W. E., Milich, R., \& Dixon, J. (1992). Single and combined effects of methylphenidate and behavior therapy on the classroom performance of children with attention-deficit hyperactivity disorder. Journal of abnormal child psychology, 20(2), 213232.

Freilich, R., \& Shechtman, Z. (2010). The contribution of art therapy to the social, emotional, and academic adjustment of children with learning disabilities. The Arts in Psychotherapy, 37, $97-$ 105

Dalebroux, A., Goldstein, T. R., \& Winner, E. (2008). Short-term mood repair through art-making: Positive emotion is more effective than venting. Motivation and Emotion, 32(4), 288-295.

Drake, J. E., Coleman, K., \& Winner, E. (2011). Short-term mood repair through art: Effects of medium and strategy. Art Therapy, 28(1), 26-30.

Epp, K. M. (2008). Outcome-based evaluation of a social skills program using art therapy and group therapy for children on the autism spectrum. Children \& Schools, 30(1), 27-36.

Gilliam, J.E. (1995). The Attention-Deficit/Hyperactivity Disorder Test: A method for Identifying $A D H D$. Austin, TX: PRO-ED. 
Henley, D. (1998). Art therapy in a socialization program for children with attention deficit hyperactivity disorder. American Journal of Art Therapy, 37(1), 2-11.

Jackson, N. A. (2003). A survey of music therapy methods and their role in the treatment of early elementary school children with ADHD. Journal of Music Therapy, 40(4), 302-323.

Kearns, D. (2004). Art therapy with a child experiencing sensory integration difficulty. Art Therapy, 21(2), 95-101.

Klein, R. G., \& Abikoff, H. (1997). Behavior therapy and methylphenidate in the treatment of children with ADHD. Journal of Attention Disorders, 2(2), 89-114.

Koppitz, E. M. (1968) Psychological evaluation of children's human figure drawings. New York: Grure \& Stratton.

Kozlowska, K., \& Hanney, L. (2001). An art therapy group for children traumatized by parental violence and separation. Clinical Child Psychology and Psychiatry, 6(1), 49-78.

Malchiodi, C. A. (2003). Art therapy and the brain. In C. A. Malchiodi (Ed.), Handbook of art therapy (pp. 16-24). New York: Guildford.

Mousavi, M., \& Sohrabi, N. (2014). Effects of art therapy on anger and self-esteem in aggressive children. Procedia-Social and Behavioral Sciences, 113, 111-117.

MTA Cooperative Group. (1999). A 14-months randomized clinical trial of treatment strategies for attention-deficit/hyperactivity disorder. Archives of General Psychiatry, 56, 1073-1086.

Pliszka, S. R. (2009). Treating ADHD and comorbid disorders: Psychosocial and psychopharmacological interventions. Guilford Press.

Safran, D. (2002). Art therapy and AD/HD: Diagnostic and therapeutic approaches. USA: Jessica Kingsley Publishers.

Schweizer, C., Knorth, E. J., \& Spreen, M. (2014). Art therapy with children with autism spectrum disorders: A review of clinical case descriptions on 'what works'. The Arts in Psychotherapy, $41,577-593$

Safran, D. S. (2003). An art therapy approach to attention-deficit/hyperactivity disorder. In C. A. Malchiodi (Ed.), Handbook of art therapy (pp. 181-192). New York: Guildford.

Slayton, S. C., D'Archer, J., \& Kaplan, F. (2010). Outcome studies on the efficacy of art therapy: A review of findings. Art Therapy, 27, 108-118.

Smitheman-Brown, V., \& Church, R. R. (1996). Mandala drawing: Facilitating creative growth in children with ADD or ADHD. Art Therapy, 13(4), 252-260.

Van de Vijfeijken, K. (1994). Normering menstekening voor 4-12 jarige kinderen [HFD norms for 412 year old children]. Leiden: Leiden University Press.

Waller, D. (2006). Art therapy for children: How it leads to change. Clinical Child Psychology and Psychiatry, 11(2), 271-282.

Webster-Stratton, C., Reid, J., \& Hammond, M. (2001). Social skills and problem-solving training for children with early-onset conduct problems: Who benefits?. Journal of Child Psychology and Psychiatry, 42(07), 943-952. 